\title{
Construcción y validación de un instrumento para valorar la calidad de la formación de profesores de Educación Física en Chile Construction and validation of an instrument for assessing the quality of the training of teachers of physical education in Chile \\ *Marcelo González Orb, *Edgardo Molina Sotomayor, *Mariela Ferreira Urzúa, *Roberto Leiva Contardo, ${ }^{* *}$ Cristian Martínez Salazar, *Héctor Trujillo Galindo, ***Manuel Lobos González,****Ana Beltrán González, *****Rodrigo Vargas Vitoria, ******Jorge Flandez Valderrama, $* * * * * * *$ Mario Negrón Molina \\ *Universidad Metropolitana de Ciencias de la Educación, **Universidad de La Frontera Temuco, ***Universidad Nacional Andrés

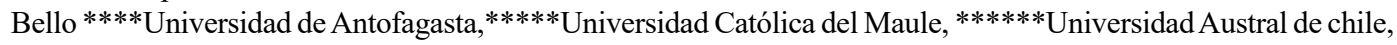 $* * * * * * *$ Universidad de los Lagos
}

Resumen. El déficit de instrumentos para valorar la calidad de formación otorgada a los alumnos de la carrera de pedagogía en educación física, a partir de la determinación de fortalezas y debilidades, antes del egreso, asociada al conocimiento de las bases curriculares y planes y programas de la asignatura de Educación Física y Salud, del Ministerio de Educación de Chile, limita de una u otra forma introducir remediales anticipadamente. El presente es un estudio descriptivo, proyectivo transversal, contempló la determinación de protocolos de elaboración (consulta de expertos nacionales $(n=8)$ e internacionales $(n=6)$ respecto a aspectos formales, metodológicos y selección de ítems por dimensión (Expresión, Didáctica, Gestión, Entrenamiento, Deportes, Recreación, Salud y Primeros Auxilios). En su validación participaron alumnos (as) del último año de la carrera. La muestra $N=214, n=133$ hombres y $n=81$ mujeres, de seis entidades de educación superior. Los resultados permiten determinar que el instrumento en su primera versión de 90 ítems presenta una consistencia interna de .756, un índice promedio de dificultad adecuada de 0,54, un índice promedio de discriminación bueno de 0,35 y un coeficiente de discriminación regular de 0,21 . Hechas las correspondientes correcciones a partir de la evidencia, se configuró una versión final del instrumento con 70 ítems, cuya caracterización psicométrica presenta una consistencia interna de .778, un índice promedio de dificultad adecuada de 0,59 , un índice promedio de discriminación alta de 0,39 y un coeficiente de discriminación bueno de 0,25 , lo que permite señalar que la prueba es adecuada para medir adquisición de saberes en el proceso de formación.

Palabra clave: Calidad de Formación; Instrumento de evaluación; Innovación educativa, formación inicial del profesor de educación física; conocimiento disciplinar.

Summary. The lack of instruments to establish the quality of training given to the students of the career of pedagogy in physical education, starting from the determination of strengths and weaknesses, before the exit, associated to the knowledge of the curricular bases and Plans and programs of the subject of physical education and health, of the Ministry of Education of Chile, limits one way or another to introduce remedial in advance. This is a descriptive, projective transversal study, contemplated the determination of elaboration protocols (consultation of national experts $(n=8)$ and international $(n=6))$ With respect to formal, methodological aspects and selection of items by dimension ( expression, didactics, management, training, sports, recreation, health and first aid). In their validation participated students of the last year of the race. The sample $n=214, n=133$ men and $n=81$ women, of six higher education institutions. The results make it possible to determine that the instrument in its first version of 90 items presents an internal consistency of. 756, an average rate of adequate difficulty of 0.54 , an average rate of good discrimination of 0.35 and a coefficient of discrimination Regular 0.21 . made the corresponding corrections from the evidence, a final version of the instrument was configured with 70 items, whose psychometric characterization has an internal consistency of. 778, an average adequate difficulty rate of 0.59 , an index An average of 0.39 high discrimination and a good discrimination coefficient of 0.25 , which makes it possible to point out that the test is suitable for measuring knowledge acquisition in the training process.

Keywords. Quality of Formation; Instrument of evaluation; educational Innovation, initial formation of the teacher of physical education; knowledge to discipline.

\section{Introducción}

En Chile actualmente no existen instrumentos validados que permitan determinar el nivel de conocimiento de los futuros profesionales, profesores Educación Física, respecto a los sectores de aprendizaje del currículum nacional de la especialidad que consideren los nuevos planes y programas del Ministerio de Educación de Chile (Mineduc).

Los cambios introducidos por la Reforma Educacional en cuanto al quehacer pedagógico de los docentes y los contenidos a desarrollar en las aulas a nivel escolar, necesariamente lleva a preocuparse por el tema de su evaluación, a partir del currículum de la especialidad de educación física,

\footnotetext{
Fecha recepción: 20-12-18. Fecha de aceptación: 06-09-19 Marcelo González Orb
}

smgorb@yahoo.es ello en base a los aprendizajes esperados, que operan como estándares en los distintos niveles educativos, de tal manera de la necesidad de elaborar un instrumento de medición que permita establecer el nivel de dominio de los contenidos de los futuros docentes egresados de las universidades, de la especialidad de pedagogía en educación física.

Lo anterior lleva a despejar algunas interrogantes respecto al sector de aprendizaje: - ¿Qué aprenden los alumnos que estudian pedagogía en educación física?, ¿Cuál es el nivel de dominio de los aprendizajes?, ¿Qué competencias han desarrollado en base de esos aprendizajes?

$\mathrm{Al}$ respecto (Urrejola \& Williams, 2017), considera que existe una relación directa entre la calidad de un instrumento de evaluación y la capacidad de los administradores de la educación para tomar decisiones educativas válidas, es decir, un instrumento de evaluación dudosa calidad, puede hacer más daño, cuando los resultados de la evaluación se utilizan 
para la formulación de políticas educativas. Por lo tanto, a partir del desarrollo de competencias profesionales establecidas, como resultado de exigencias esperadas por los organismos técnicos que formulan las bases curriculares, emanadas del Ministerio de Educación, determinan establecer cuanto saben y dominan los futuros docentes antes del egreso (Ávalos, 2014), (Serrano, 1989).

La construcción de un instrumento que permita recabar información asociada a la calidad del proceso formativo llevado a cabo al interior de las entidades de la formación de los cuadros académicos que orientan dicha formación, así como el establecer cómo se llevan a cabo dicho aprendizaje, aspecto de suma importancia, pues se han de revisar tanto los procesos formativos, es decir, revisar tanto la evolución de los planes de estudio de Educación Física, aspecto destacado por (Pedraz, Paz, \& Polo, 2014) con motivo de actualización-vigencia, aspecto determinante para avanzar en la mejora educativo-formativa, estando en concordancia con los propósitos de cada programa de formación, pues a partir de ello será la transferencia al medio escolar, teniendo presente la importancia de la innovación en la formación del profesorado, pues de esta forma la educación física avanzará y captará más adeptos, debido al reconocimiento de su práctica, aspecto destacado por (Carreiro da Costa, Valeiro, \& Villalobos, 2016), (González Tirados \& González Maura, 2007) para quienes la innovación en la formación del profesorado de educación física, adquiere un protagonismo importante, lo que medita contar con indicadores de actualización, con el propósito de garantizar de una u otra manera la calidad de formación otorgada.

En este sentido, es fundamental contar con instrumentos y protocolos rigurosos de construcción y aplicación, en función de las consecuencias implicadas respecto a la información que ésta pueda reportar, es decir, su utilidad radica en servir en la determinación del cumplimiento de los objetivos para los cuales es diseñada, respetando para ello los estándares asociados para recabar dicha información (Pedraja-Rejas, Araneda-Guirriman, Rodríguez-Ponce, \& Rodríguez-Ponce, 2012), (Sebastiani, Solá, Capell, Campos, \& Blásquez, 2009), (Garbanzo, 2007), entre otros.

En este caso, el instrumento tiene como propósito de establecer el grado de conocimiento de los futuros profesionales de la actividad fisca, respecto al dominio de los contenidos de las bases curriculares y los planes y programas, vigentes al año 2017, de la signatura de Educación Física y Salud, emanados del Ministerio de Educación (Mineduc), a objeto de garantizar la calidad educativa (Lemaitre, Maturana, Zenteno, \& Alvarado, 2012),(Ruffnelli, 2014),(Rufinelli, 2011) y la vigencia, siendo la formación permanente una herramienta para mejorar la intervención del maestro de educación física, aspecto destacado por (López, García, España, \& Barco, 2018).

Se debe tener presente, que la necesidad de la mejora de los desempeños de los profesores en el sistema escolar y universitario, lleva poner acento en la calidad de la formación inicial docente, en la evaluación del desempeño docente, y en el aseguramiento de la calidad aspecto destacado por (González \& Espinoza, 2008), en referencia a lo establecido en la Ley 20,129 y en (Norma, Materia, \& Municipal, 2016), el cual reglamenta los procesos de evaluación de competen- cias de los profesores recién egresados, el reglamento sobre evaluación docente orientado a fortalecer la profesión, favoreciendo el reconocimiento de las fortalezas y la superación de las debilidades, (Imbernon, 1989), otros documentos que emanan del Mineduc, y otros que han sido de preocupación de otros autores dentro de los cuales destacan: (Fernández Cruz, Rodríguez Mantilla, \& Fernández Díaz, 2015), (Boned Pascual, Rodriguez Romo, MAyorga GArcia, \& Alvaro, 2013; Ruffinelli, 2014; Sebastiani et al., 2009),(Sevillano, 2011), (Cecilio \& Yanes, 2018),(De-Juanas, 2011), (Santelices Etchegaray \& Valenzuela Rojas, 2015) entre otros, quienes han planteado la importancia del dominio de las competencias profesionales, para resolver situaciones teóricas-prácticas durante el desempeño, competencias que han de ser adquiridas durante la formación inicial del profesorado de educación física (Cañadas, Santos-pastor, \& Castejón, 2019) lo cual es reafirmado también a través del tiempo por (Vaillant, 2008),(Del, En, \& Jaña, 2005; Miranda Jaña \& Rivera Rivera, 2009).

Es preciso destacar que se ha avanzado con la promulgación de La Ley N¹9.961 (Mineduc 2004), sobre evaluación docente, algunos autores que han influenciado la discusión son María José Lemaitre, Mario Maturana, Elisa Zenteno, Andrea Alvarado, (2012). Se debe tener presente que la responsabilidad recae en las universidades, lo cual exige otorgar una preparación adecuada, debiéndose revisar los procesos formativos, comenzando por la autorreflexión de quehacer profesional, como lo proponen Pedraja-Rejas, Liliana M, Araneda-Guirriman, Carmen A, Rodríguez-Ponce, Emilio R, \& Rodríguez-Ponce, Juan J. (2012), ante lo cual el punto de partida, debe ser la propia autoevaluación de dominios y debilidades, (Concepciones et al., 2008).

Las universidades han de asumir su rol, transfiriendo aprendizajes a los futuros profesores y estos a los educandos, para transformar la sociedad, a partir de la práctica en las unidades educativas y del otorgar una formación de excelencia,(Carmen Paez \& Hurtado Almonacid, 2019), (Tobón, Rial Sánchez, Carretero, \& García, 2014) teniendo presente para su éxito que los docentes universitarios, deben aportar conocimientos pedagógicos y disciplinares, que permitan a los futuros profesionales de la educación ser de excelencia, preocupación surgida desde hace algún tiempo pero vigente en la actualidad, como lo manifiestan (Villarroel \& Bruna, 2017), (Pedraja-Rejas et al., 2012), (Rodríguez Martínez, 2014), (Feu \& Carrizosa, 2018) entre otros.

Una de las etapas importante del proceso, lo constituye el diseño de una prueba que permita dar cuenta de la calidad de formación otorgada, el cual debe definir aspectos técnicos que van desde el determinar la formulación de dimensiones, la elaboración de preguntas, establecer protocolos de validación, aplicación y de corrección.

Acorde a lo señalado, se han de definir en primer lugar los propósitos de aplicación del instrumento-prueba, los alcances de sus resultados, proyecciones e impactos en el proceso formativo, este último aspecto es destacado por (Feu \& Carrizosa, 2018) quien señala la importancia de contar con instrumentos validados para medir el Conocimiento Didáctico del Contenido en el profesorado de Educación Física, así como otros de singular importancia, en tal sentido el estipular el qué medir, para qué, a quién y cómo, son aspec- 
tos importantes y fundamentales al momento de su elaboración.

A partir de las interrogantes planteadas, el instrumento se orienta de una u otra manera, a certificar qué se ha aprendido, durante el proceso de aprendizaje, respecto a lo esperado como dominio disciplinar, durante los años de duración de la carrera, ante lo cual se ha de contar con instrumentos y procedimientos para establecer su calidad, (Carrillo \& Valenzuela, 2012; De Diego, M. y Rueda, 2012; De La Orden, 2009; Fernández Cruz et al., 2015; Montecinos, Rittershaussen, Solís, Contreras, \& Iglesias, 2010), con motivo de mejorar la calidad de formación universitaria.

\section{Metodología}

\section{Diseño investigativo}

Estudio de diseño cuantitativo, de carácter descriptivo, orientado a validar un instrumento orientado determinar la calidad de formación universitaria, otorgada en los contextos de la espacialidad de educación física, en sus protocolos se respetaron las consideraciones de (Briones, Colombiano, \& El, 2002; Hernández, Fernández, \& Baptista, 2014).

\section{Muestra}

La caracterización psicométrica se realizó en seis universidades chilenas, muestra aleatoria, conformada por 214 sujetos (133 hombres y 81 mujeres), sujetos voluntarios convocados a participar en su condición de estudiantes del último año de la carrera de pedagogía y licenciatura en educación física. (Ver Tabla. 1), caracterización de la muestra por entidad participante, en su selección se contemplaron las sugerencias de (González \& Espinoza, 2008; Hernández et al., 2014; Otzen \& Manterola, 2017).

\begin{tabular}{|c|c|c|c|c|c|c|}
\hline \multicolumn{7}{|c|}{ a } \\
\hline & & \multicolumn{2}{|c|}{ Hombre } & \multicolumn{2}{|c|}{ Mujer } & \multirow{2}{*}{$\begin{array}{l}\text { Total } \\
\mathrm{N}\end{array}$} \\
\hline & & $\mathrm{N}$ & $\%$ & $\mathrm{~N}$ & $\%$ & \\
\hline \multirow{7}{*}{ Universidad } & $\begin{array}{l}\text { Universidad de } \\
\text { Antofagasta }\end{array}$ & 11 & $55,0 \%$ & 9 & $45,0 \%$ & 20 \\
\hline & UMCE & 18 & $51,4 \%$ & 17 & $48,6 \%$ & 35 \\
\hline & UC Maule & 39 & $66,1 \%$ & 20 & $33,9 \%$ & 59 \\
\hline & UFRO & 18 & $62,1 \%$ & 11 & $37,9 \%$ & 29 \\
\hline & U Austral & 22 & $57,9 \%$ & 16 & $42,1 \%$ & 38 \\
\hline & U Los Lagos & 25 & $75,8 \%$ & 8 & $24,2 \%$ & 33 \\
\hline & Total & 133 & $62,1 \%$ & 81 & $37,9 \%$ & 214 \\
\hline
\end{tabular}

Se debe tener presente que el instrumento aplicado fue sometido a consideraciones éticas, aprobadas por el comité de Ética Institucional de la Universidad de Santiago de Chile, acta que consta en el informe ético $\mathrm{N}^{\circ}$ : 334, del año 2017, el cual se presentó en cada una de las entidades participantes, informando a los estudiantes de la muestra el propósito de la investigación, explicando los protocolos de aplicación, de alcances del estudio y resguardo de la información, solicitando su participación voluntaria mediante consentimiento informado de participación, garantizando a estos la confidencialidad y la protección de su identidad.

\section{Protocolo elaboración Instrumento}

En el proceso de elaboración la Prueba de Conocimientos Específicos, se respetaron las propuestas formativas a partir de los planes y programas de la especialidad de Educación Física. El objetivo de este se orienta a estimar el nivel de conocimiento, al término del proceso de formación de la es- pecialidad, respecto a los dominios de los contenidos de la Asignatura de Educación Física y Salud, asociada a las bases curriculares y los programas emanados del Ministerio de Educación de Chile (MINEDUC).

Está conformado de siete dimensiones o áreas de conocimiento formativas: Expresión, Didáctica, Gestión, Entrenamiento, Deportes, Recreación y Salud y Primeros Auxilios, las cuales son definidas a partir de la revisión del currículum nacional, con un total de 70 ítems, aspecto que es analizado de forma precedente.

\section{Validación de contenido}

La validez de contenido se asocia de una u otra manera con la determinación del muestreo a emplear en la elaboración de una prueba, frente al universo de posibles conductas y o conocimientos que se pretenden medir (Cohen y Swerdlik, 2001, citados por (Escobar-Pérez \& CuervoMartínez, 2008)), (Andreú, 2002) en tal sentido la primera etapa del protocolo, contemplo la enunciación de los reactivos, los cuales fueron formulados a partir de expertos de cada una de las espacialidades y dimensiones contempladas en el instrumento, pertenecientes a las universidades Antofagasta, Austral de Chile, Católica del Maule, Central de Chile, Frontera de Temuco, Los Lagos y Metropolitana de Ciencias de la Educación, para ello se respetaron las sugerencias de (Urrutia Egaña, Barrios Araya, Gutiérrez Núñez, \& Mayorga Camus, 2015). La segunda etapa, de análisis de ítems, fue desarrollado por expertos por especialidad, todos académicos-profesionales asociados al área de estudio en Educación Física, de reconocida trayectoria académica en sus respectivos campos de especialidad, con experiencia en la determinación de competencias en pertenecientes a la Universidad Metropolitana de Ciencias de la Educación, los que tuvieron a la vista documentos afines para la determinación de competencias a nivel universitario y escolar, dentro de los cuales destacan (Coll, 2007; Larraín U. \& Gonzalez F., 2005),(Boned Pascual et al., 2013), (Carrillo \& Valenzuela, 2012).

La tercera etapa, revisión final de ítems, desarrollada por académicos expertos nacionales $(\mathrm{n}=8)$ e internacionales $(\mathrm{n}=6)$, con reconocida experiencia en materias asociadas a la investigación educacional, teniendo como requisito contar con experiencia disciplinar en el campo de la especialidad, con el objetivo de revisar ítems, asignar asignación de reactivo a la dimensión, según exigencia de las bases curriculares y los planes y programas de Educación Física, proceso que dio lugar a acoger sugerencias y otros aspectos asociados a la elaboración final del instrumento. (Andreú, 2002; Bolívar, 1993; Montecinos et al., 2010).

Cuarta etapa, revisión final de ítems por los expertos disciplinares nacionales, etapa, orientada a analizar sugerencias de expertos nacionales e internacionales, determinar la asignación de los ítems a dimensiones, antes de la aplicación de la prueba piloto.

Quinta etapa, última fase del proceso de validación de contenido aplicación prueba piloto, a un grupo de estudiantes de la especialidad del último año de carrera, hombres y mujeres de la Universidad Central de Chile, proceso, asociado, con fin de determinar entre otros aspectos, nivel de comprensión de los ítems, establecer aspectos de redacción, de 
contenido, establecer la opinión de aspectos formales instrumento, comprensión de instrucciones, determinación tiempos de contestación, comportamiento de ítems, protocolos de aplicación y corrección, etapa que se desarrolló aplicando las sugerencias de (Caso-Niebla \& Hernández-Guzmán, 2007; Hernández et al., 2014; Rosas et al., 2011).

Del total de reactivos, y luego de la revisión de los expertos, de acoger recomendaciones, del proceso de la prueba piloto y siguiendo algunas de las recomendaciones de (Escobar-Pérez \& Cuervo-Martínez, 2008) se conforma el instrumento original con de 90 reactivos de opción múltiple de cuatro alternativas, referidas a siete áreas formativas: Expresión, Didáctica, Gestión, Entrenamiento, Deportes, Recreación y Salud y primeros auxilios. (Ver Tabla. 2).

\begin{tabular}{lcc}
$\begin{array}{l}\text { Tabla. } 2 \\
\text { Determinación items por dimensión, instrumento 1 }\end{array}$ & & \\
\hline Dimensión & Items & $\%$ \\
\hline 1. Expresión & 7 & 7,7 \\
2. Didáctica & 14 & 15,5 \\
3. Gestión & 9 & 10 \\
4. Entrenamiento & 19 & 21,1 \\
5. Deportes & 18 & 20 \\
6. Recreación & 5 & 5,5 \\
7. Salud y PP AA & 18 & 20 \\
Total instrumento & 90 & 100 \\
\hline
\end{tabular}

\section{Protocolo aplicación prueba final}

La Prueba de Conocimientos Específicos Bases y Programas de la especialidad de Educación Física, fue aplicada previa preparación de jueces académicos respetando las sugerencias de (Urrutia Egaña, Barrios Araya, Gutiérrez Núñez, \& Mayorga Camus, 2015) con motivo de uniformar su administración, siguiendo protocolos similares de trabajo de campo, en cada entidad. Previo a las instrucciones de su contestación, se dieron a conocer, los alcances del estudio, los protocolos de administración y contestación, entregando a los participantes los consentimientos informados, leyendo uniformemente instrucciones, se disiparon dudas, a la totalidad de los asistentes, se definió el tiempo de administración correspondiendo a 120 minutos, para la totalidad de las universidades participantes.

Se debe desatacar que los datos de caracterización de la muestra y de respuestas a los reactivos fueron registrados en una hoja de respuesta del software PC-OMR versión 8, para su traspaso a formato digital de análisis.

\section{Análisis estadísticos}

Se efectuaron análisis de confiabilidad mediante el Índice Alpha de Cronbach del instrumento total y se calcularon para cada dimensión, el índice de dificultad, índice de discriminación y el coeficiente de discriminación a través del coeficiente de correlación del punto biserial, mediante el paquete estadístico Stastical Package for the Social Sciences (SPSS) versión 20., lo cual permitió establecer los aspectos asociados al rendimiento.

A partir de los resultados obtenidos y de los correspondientes análisis, se desarrollan los últimos ajustes que dan finalmente como producto un instrumento revisado.

\section{Resultados}

Estos se exponen a continuación determinando en primer lugar la consistencia interna del instrumento tanto en términos generales, como específicos por dimensión, los que son presentados mediante estadísticos descriptivos, expresados en porcentajes de logro, expuestos en las siete dimensiones y en términos generales, para la muestra total y por sexo, caracterizados de forma psicométrica por nivel de dificultad y discriminación.

Consistencia interna de la prueba, considerando la totalidad de los 90 ítems del test, obtenida a través de Alpha de Cronbach es de 0,756, la que, según DeVellis (1991) puede ser tipificada como respetable, coincidente con lo planteado por Murphy y Davishofer (en Hogan, 2004) como confiabilidad moderada.

Analizado los niveles de dificultad de la prueba, en general y por dimensiones, se puede señalar que los ítems presentan grados de dificultad (p) adecuados, entre medianamente difíciles y medianamente fáciles. (Ver (Tabla. 5).

Para analizar los niveles de discriminación del instrumen-

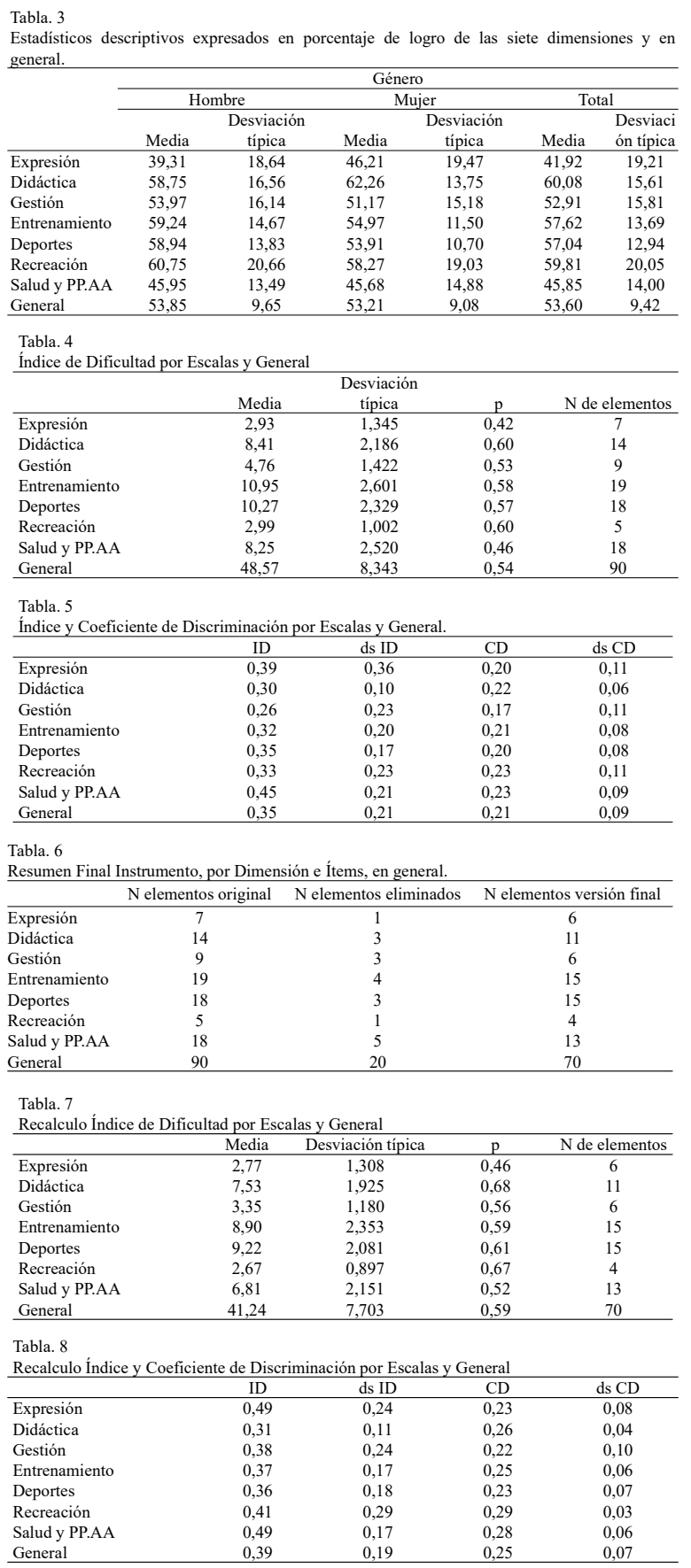


to, es decir, determinar si quien tuvo una puntuación alta en toda la prueba debería tener altas probabilidades de contestar correctamente el ítem, se utilizó el índice de discriminación y el coeficiente de discriminación. La diferencia de uso entre el coeficiente de discriminación y el índice de discriminación (D) es que con el primer método se toman en cuenta todas y cada una de las personas evaluadas, mientras que, con el segundo, sólo se toma $54 \%$ de ellas (27\% más alto y $27 \%$ más bajo). Mientras más alto es el índice de discriminación, el reactivo diferenciará mejor a las personas con altas y bajas puntuaciones.

Según estándares internacionales la distribución de los resultados del coeficiente de discriminación basada en correlación Biserial Puntual es entre pobre y regular y alta según el índice de discriminación, motivo por el cual se debe hacer una revisión y eliminación de ítems para una segunda versión del instrumento, principalmente aquellos que discriminan de modo inverso.

A partir de los análisis informados de consistencia interna de la prueba, del índice de dificultad, índice de discriminación y el coeficiente de discriminación, se ha decidido eliminar 20 ítems del instrumento de acuerdo al detalle: (Ver Tabla $6)$.

La consistencia interna del instrumento se mantiene moderada con un valor Alpha de Cronbach de 0,778

$\mathrm{Al}$ recalcular el índice de dificultad por dimensión y en general, se eleva levemente hacia medianamente fácil. (Ver Tabla. 7).

Como se puede observar en la Tabla 8 , al eliminar los ítems con discriminación inversa, nula o baja, se presentan niveles altos en este índice y coeficiente, como se muestra a continuación. (Ver Tabla. 8).

\section{Discusión}

El objetivo principal de este estudio fue construir y validar un instrumento que permita establecer el dominio de los contenidos disciplinares asociados a los Planes y Programas establecidos por el (Mineduc), en los futuros docentes egresados de seis universidades chilenas, en la Licenciatura y Pedagogía en Educación Física.

Es importante destacar la importancia de contar con instrumentos validados para medir el Conocimiento Didáctico del Contenido en el profesorado de Educación Física, esto permite indagar en las fuentes de conocimiento base para la docencia que deben presentar los futuros profesores de educación física. En este caso, el instrumento inicial consideró el conocimiento del contenido de la materia enseñada con un total de 90 ítems, distribuidos en 7 dimensiones.

Para el diseño y validación se utilizó un panel de 15 jueces expertos que para Garcia y Fernandez (2008) es la cantidad ideal de expertos. El análisis del panel de expertos permitió profundizar y analizar de forma apropiada cada uno de los ítems diseñados quienes contribuyeron tanto en aspectos cuantitativas, como cualitativas que permitieron perfeccionar el instrumento, siendo esta metodología ampliamente utilizada para validar el contenido de los instrumentos de evaluación. (Messick, 1989),(Carpio de los Pinos et al., 2013), (Feu \& Carrizosa, 2018; García, Fernández, Terrón, \& Blanco, 2008).
Finalizada la etapa de validación del instrumento por los jueces expertos, se redactó el instrumento definitivo que fue aplicado a una muestra de estudiantes egresados de Licenciatura y Pedagogía en Educación Física de siete universidades chilenas (muestra de 214 estudiantes).

Para determinar la fiabilidad y capacidad de reproductividad del instrumento tanto en términos generales como específicos por dimensión se presentaron los resultados con estadísticos descriptivos, expresados en porcentajes de logro, expuestos en las siete dimensiones y en términos generales, para la muestra total y por sexo, caracterizados de forma psicométrica por los niveles de dificultad y discriminación. Los resultados indican que el índice de dificultad y discriminación del instrumento por dimensión y en general, mostro grados de dificultad (p) adecuados, entre medianamente difíciles y medianamente fáciles. (Ver Tabla. 5).

Utilizando los valores $\mathrm{D}$, se puede observar que los ítems que discriminan en forma excelente con valor de 0,45 son los asociados al dominio de Salud y PPAA. Los ítems de los dominios de Expresión, Didáctica, Entrenamiento, Deportes, y Recreación presentaron un buen poder discriminativo con valores de 0,30 a 0,39. Sin embargo, el dominio de gestión presentó los valores más deficientes de todos los dominios. Por otro lado, el coeficiente de discriminación, muestra que el dominio de Gestión presenta un débil nivel discriminativo con un valor de 0,17 a diferencia de los dominios restantes que muestran un buen poder discriminativo.

En base a todos estos resultados y de acuerdo a estándares internacionales la distribución del coeficiente de discriminación basada en correlación Biserial Puntual indicó que los dominios eran pobre y regular y alta según el índice de discriminación, lo cual llevó a una segunda versión del instrumento, eliminando 20 ítems que discriminan de modo inverso. Es importante mencionar que los mejores indicadores de la efectividad discriminativa de un reactivo son el punto de correlación biserial y el coeficiente de correlación biserial, a los cuales se les llama coeficientes de discriminación (Rosas Morales, BackhoffEscudero, \& Larrazolo Reyna, 2000).

Por otro lado, también se evaluó la consistencia interna del instrumento utilizando para ello el estadístico del Alfa Cronbach, que revela el grado de covarianza de los items que configuran el instrumento, lo cual constituye un indicador de consistencia interna (Feu \& Carrizosa, 2018). En base a este estadístico es posible afirmar que la consistencia interna inicial de éste fue aceptable, ya que el valor fue de $0,756 \mathrm{y}$ al eliminar aquellos ítems que discriminaban negativamente la segunda versión obtuvo un valor de 0,778 , esto sugiere que el instrumento permite medir las dimensiones del conocimiento del contenido disciplinario propuestos en este instrumento, lo que es confirmado tanto por (Flores-Ruiz et al., 2017) y DeVellis (1991), siendo tipificada como respetable, coincidente con lo planteado por Murphy y Davishofer (en Hogan, (2004)) como confiabilidad moderada (Ver Tabla. 3). Al eliminar los ítems con discriminación inversa, nula o baja se puede observar en la tabla 7 y 8 como aumentan el índice de dificultad, índice de discriminación y coeficiente de discriminación.

En relación a los resultados obtenidos, y efectuados los ajustes correspondientes, asociados a la optimización del 
instrumento, se puede señalar que éste constituye un aporte para establecer el nivel de formación desarrollado al interior de las carreras de Licenciatura y Pedagogía en Educación Física, aspecto de exigencia nacional y que a la fecha del desarrollo de la propuesta, se carecía en Chile, es decir, instrumentos de medición que permitan contar con la apreciación de dominio y conocimiento de los futuros profesionales de la educación física, respecto a los sectores de aprendizaje del currículum nacional de la especialidad, lo que concuerda con los estudios desarrollados por (Vázquez, Biggio, \& García, 2013)

Por otra parte, las exigencias de las reformas educacionales respecto al quehacer pedagógico de los futuros docentes, demanda establecer los dominios de los contenidos a desarrollar a nivel escolar, aspecto que debe estar asociado a partir del currículum de la especialidad de educación física, es decir, los aprendizajes esperados, como estándares, ante ello el aportar instrumento y protocolos de medición para establecer el nivel de dominio de los contenidos por parte de los futuros docentes egresados constituye todo un aporte, pues permiten despejar las interrogantes respecto a ¿Qué aprenden los alumnos que estudian pedagogía en educación física?, ¿Cuál es el nivel de dominio de los aprendizajes?, ¿Qué competencias han desarrollado en base de esos aprendizajes?, a partir del instrumento propuesto, pero teniendo presente, que estos resultados y las medidas a implementar en su mejora, han de ser específicos en cada realidad formativa, pues estas presentaran fortalezas y debilidades propias, las que han de ser superadas acorde a los resultados específicos obtenidos, implementando protocolos en función a la realidad de recursos disponibles, herramientas paliativas al alcance, de la problemática manifestada.

Se debe tener presente que el instrumento propuesto, debe ser actualizado en cada oportunidad que sea aplicado, pues los planes y programas en términos generales, sufren constantes modificaciones y adecuaciones en el tiempo, en tal sentido sus contenidos, han de estar contextualizados en función de las exigencias establecidas, si adquieren singular importancia los protocolos de elaboración, tanto de los ítems, como las dimensiones propuestas en cada oportunidad, ello acorde a lo señalado anteriormente, actualización o modificaciones de bases curriculares, o reformas educacionales del campo de la especialidad, como de los planes y programas según corresponda (Andreú, 2002).

En relación a los resultados obtenidos, y producto de los ajustes y reajustes, efectuados, al eliminar algunos reactivos, el instrumento adquirió mayor nivel de fiabilidad, respetando las sugerencias de (Hernández et al., 2014), lo que lo hace apropiado para ser aplicado en distintas realidades formativas, de acuerdo a lo plantado por otros autores que proponen que para disminuir el factor error, cada dimensión debe contar con un número superior de ítems, ante lo cual se han de reforzar aquellos que presenten un número escaso de éstos (Urrutia Egaña et al., 2015).

\section{Conclusiones}

En función a los resultados obtenidos por los alumnos en la prueba de conocimientos específicos de la especialidad de educación física, es posible concluir:

Los alumnos han obtenidos resultados tanto en términos generales como específicos, bajo lo normal esperado, lo cual que permiten señalar que la prueba es capaz de identificar los dominios tanto por dimensión como en términos globales.

En cuanto a las dimensiones establecidas, estas permiten establecer las áreas de fortaleza como de debilidad, lo que da lugar a introducir mejoras al proceso formativo acorde a cada realidad universitaria.

El análisis por ítems da lugar a identificar los dominios adquiridos y aquellos que han de ser revisados, a objeto de garantizar la calidad de la formación otorgada, a los alumnos a nivel de la espacialidad.

Los análisis por dimensión, permite determinar que se han de incluir algunos ítems a algunas dimensiones, con motivo de identificar con mayor asertividad el dominio adquirido.

En referencia al comportamiento general del instrumento, y acorde a los resultados obtenidos en su aplicación en las distintas entidades de educación superior participantes en el estudio, presenta estabilidad, dando lugar a recomendar su aplicación en otras realidades formativas de la espacialidad.

En relación al número de ítems por dimensión, es posible señalar que en términos generales este cumple plenamente con el objetivo de identificar las áreas débiles de promoción, aun cuando algunas hacen necesario incorporar algunos ítems para otorgar un mayor peso a la dimensión.

En relación a la determinación del dominio de la competencia, se sugiere dividir el instrumento en dos mitades, acogiendo el protocolo de instrumentos de mitades partidas, la primera de carácter de dominio de los conocimientos teóricos y la otra mitad de conocimientos aplicativos.

En referencia a la extensión del instrumento, este demuestra apropiado en su extensión, ello avalado en la opinión de los alumnos (as) participantes en el estudio.

En referencia a la metodología de análisis aplicada, esta e presenta adecuada a la naturaleza del estudio, dado que permite identificar tanto las fortalezas como debilidades de la formación, tanto en aspectos específicos, por dimensión como en términos globales de necesidades formativas.

En cuanto a las características del instrumento es posible señalar que este permite clasificar las preguntas de acuerdo a cada dimensión y a partir de estas, realizar el análisis de clasificación de los ítems, con fin de establecer su equivalencia.

Se puede apreciar que el instrumento se centra principalmente en las áreas de Entrenamiento (21,1\%) Deportes (20\%), Salud-Primeros Auxilios (20\%) y Didáctica (15,5\%) lo que demanda un mayor dominio de la especialidad, mientras otras áreas a fines a la formación como Gestion (10\%), Expresión (7\%) y Recreación (5\%), muestran un desequilibrio en lo que se mide según el desempeño y por lo presentado en los programas. Se debe tener presente que la prueba facilita el análisis de los dominios adquiridos, pero el conjunto determina el dominio de la especialidad.

El instrumento prueba Determinación de la Calidad de la Formación de Profesores de Educación Física en Chile, permite establecer en forma objetiva el grado de dominio de los 
conocimientos por parte de los alumnos de la carrera de Pedagogía en Educación Física en cuanto a conocimiento de las bases curriculares, planes y programas emanado del Mineduc.

Estos dominios contemplan desde el inicio del proceso, es decir, de $1^{\circ}$ a $8^{\circ}$ Básico a cuarto año medio.

La prueba se presenta como un instrumento válido, que permite determinar las fortalezas y debilidades formativas a nivel de educación superior, en la espacialidad de Educación Física. Dada su detallada elaboración y los protocolos de aplicación, respaldados por los antecedentes estadísticos, permite recomendarlo, efectuando los correspondientes ajustes, en las instituciones formativas de educación superior para su utilización.

El análisis realizado da cuenta de la necesidad de optimizar el instrumento, a partir de la detección de algunos vacíos e imprecisiones en la medición, lo que permite mejorar la fiabilidad del mismo.

Se debe desatacar que el instrumento si bien permite establecer las debilidades por dimensión se debe destacar el medir los términos globales la formación otorgada.

Entre las fortalezas del instrumento, es posible destacar la medición global respecto al dominio de los conocimientos teóricos y prácticos asociados al quehacer formativo de la especialidad en educación física, acorde a lo esperado por el Mineduc, concordando respecto a lo planteado por la teoría, en relación de la importancia de actuar como predictor del dominio posterior en la trasferencia al campo de la espacialidad.

Una de sus principales debilidades del proceso formativo se asocia a la falta de aplicación de instrumentos que den garantía del dominio de los contenidos vertidos en las aulas universitarias. Esto representa la importancia de medir antes del egreso de las correspondientes carreras, determinar las fortalezas y debilidades, a efecto de introducir remediales.

La aplicación de este tipo de medidas, da lugar a anticiparse a la problemática, otorgando la oportunidad a las entidades de educación superior actuar en forma preventiva frente al deber ser del proceso formativo, evitando con ello la multiplicación de la problemática a nivel formativo escolar.

Se espera que el profesor-estudiante, comprenda a cabalidad de la importancia de aplicación y participación en el presente proceso evaluativo, como parte del rol a desempeñar en los campos educacionales. Lo anterior, por lo general no es asumido a cabalidad por los alumnos a nivel universitario, omitiendo su rol formativo educativo de la espacialidad.

El análisis de la prueba del modelo de evaluación aplicado, instrumento de medición propone criterios de evaluación que apuntan a los diversos procesos a desarrollar por los profesores de la espacialidad de educación física, están basados en las propuestas del Mineduc, y las teorías vigentes del deber ser de la educación física, fundamentalmente el orientado al campo escolar, pero también incorpora elementos relevantes para una mirada holística del desempeño del futuro profesor de la espacialidad de educación física, lo que lo sitúa en un sistema social cambiante.

Finalmente, se debe tener presente que el instrumentoprueba debe ser actualizado constantemente, así como su diseño, dando así curso a nuevos instrumentos que midan de manera integral al futuro profesor, más aún si lo que se está formando es un niño-sujeto en edad escolar.

\section{Agradecimientos}

Por el apoyo prestado en el desarrollo de la investigación denominada, «Calidad de la formación de los alumnos en carreras de pedagogía en Educación Física, en entidades Universitarias del país, conocimiento de planes y programas del MINEDUC.

Dirección de Investigación de la Universidad Metropolitana de Ciencias de la Educación (DIUMCE). Código del proyecto, FGI 23-17.

Dirección de Investigación Universidad de La Frontera de Temuco (DIUFRO). Código del proyecto, DIE17-0008.

\section{Referencias}

Andreú, J. (2002). Las técnicas de Análisis de Contenido: una revisión actualizada. Fundación Centro de Estudios Andaluces, 1-34. https://doi.org/10.2307/334486

Avalos, B. (2003). La formacion docente inicial en Chile, (October), $2-41$.

Ávalos, B. (2014). La formación inicial docente en Chile: Tensiones entre políticas de apoyo y control. Estudios Pedagógicos (Valdivia), 40(Especial), 11-28. https://doi.org/10.4067/S071807052014000200002

Bolívar, A. (1993). «Conocimiento didactico del contenido»y formacion del profesorado: el programa de 1. Shulman. Interuniversitarta de Formación Del Profesorado.

Boned Pascual, C., Rodriguez Romo, Ga., MAyorga GArcia, J., \& Alvaro, M. J. (2013). Competencias Profesionales del Licenciado en CCAFYDE. Competencias Profesionales Del Licenciado En CCAFYDE, 53, 1689-1699. https://doi.org/10.1017/ CBO9781107415324.004

Briones, G., Colombiano, I., \& El, P. (2002). Investigación Cuantitativa En Las Ciencias Sociales. Tree Physiology (Vol. 22). https:/ /doi.org/10.1088/1751-8113/44/8/085201

Cañadas, L., Santos-pastor, M. L., \& Castejón, F. J. (2019). Competencias docentes en la formación inicial del profesorado de educación física Teaching competences in physical education teacher initial training, 2041, 284-288.

Carmen Paez, J., \& Hurtado Almonacid, J. (2019). Formación inicial docente en profesores de educación física. Levantamiento de competencias específicas a partir de las necesidades del medio educativo. Retos, 35(2012), 61-66.

Carpio de los Pinos, C., Tejero González, J. M., García Díaz, V., Carpio de, los P., Tejero González, J. M., García Díaz, V., ... García Díaz, V. (2013). Análisis de los factores influyentes en un ambiente escolar con violencia y dificultades de convivencia. Revista Española de Orientación y Psicopedagogía. https:// doi.org/10.5944/reop.vol.24.num.3.2013.11249

Carreiro da Costa, F., Valeiro, M., \& Villalobos, M. (2016). Innovación en la formación del profesorado de educación física. I Innovation in teacher training on physical education. Retos: Nuevas Perspectivas de Educación Física, Deporte y Recreación, 29(29), 251-257. https://doi.org/10.1088/0953-8984/3/ 23/016

Carrillo, A. M., \& Valenzuela, B. (2012). Evaluacion de competencias docentes para la mejora de la calidad de la Educacion Superior en Mexico. Diálogo.

Caso-Niebla, J., \& Hernández-Guzmán, L. (2007). Variables que inciden en el rendimiento académico de adolescentes mexicanos. Revista Latinoamericana de Psicologia. https://doi.org/10.4090/ juee.2008.v2n2.033040

Cecilio, E., \& Yanes, C. (2018). Los puntos de vista del concepto competencia laboral, (April).

Coll, C. (2007). Las competencis en la educacion escolar/ : Algo Más Que Una Moda Y Mucho Menos Que Un Remedio. Departamento de Psicología Evolutiva y de La Educación. Facultad de Psicología. Universidad de Barcelona. https://doi.org/1131- 
$995 \mathrm{X}$

Concepciones, L. A. S., Orientan, Q. U. E., Practicas, L. A. S., Profesores, E. D. E. L. O. S., Prieto, M., \& Contreras, G. (2008) Estudios Pedagógicos ISSN/ : 0716-050X Universidad Austral de Chile.

De-Juanas, Á. (2011). Aproximaciones teóricas sobre las competencias del profesorado de Educación Superior. Tendencias Pedagógicas, (18), 233-250.

De Diego, M. y Rueda, M. (2012). La evaluación docente en educación superior: uso de instrumentos de autoevaluación, planeación y evaluación por pares. Voces y Silencios. Revista Latinoamericana de Educación.

De La Orden, A. (2009). Evaluación y calidad: análisis de un modelo. Estudios Sobre Educacion.

Del, P., En, P., \& Jaña, C. M. (2005). Aproximacion a un modelo evaluativo de la formacion permanente del profesorado en chile, 145-166.

DeVellis, R.F. (1991). Scale development: Theory and applications Newbury Park: Sage Publications, Inc.

Educacion, S. D. E. (2008). Ministerio de educacion. 9/07.

Escobar-Pérez, J., \& Cuervo-Martínez, Á. (2008). Validez De Contenido Y Juicio De Expertos: Una Aproximación a Su Utilización. Avances En Medición, 6, 27-36.

Fernández Cruz, F. J., Rodríguez Mantilla, J. M., \& Fernández Díaz, M. J. (2015). Evaluación del impacto de la aplicación del modelo de excelencia europeo (EFQM) en el clima esoolar y en la satisfacción de sus miembros. In Investigar con y para la sociedad.

Feu, S., \& Carrizosa, M. V. (2018). Validación de un cuestionario para medir el Conocimiento Didáctico del Contenido en el profesorado de Educación Física. Retos, 0(34), 132-137. Retrieved from https://recyt.fecyt.es/index.php/retos/article/view/58590

Garbanzo Vargas, M. G. (2007). Factores asociados al rendimiento académico en estudiantes universitarios, una reflexión desde la calidad de la educación superior pública. Revista Educación. https:/ /doi.org/10.15517/revedu.v31i1.1252

García, M., Fernández, L., Terrón, M., \& Blanco, Y. (2008). Métodos de evaluación para las competencias generales más demandadas en el mercado laboral. TICAI.

González, L. E., \& Espinoza, O. (2008). Calidad de la educación superior: Concepto y Modelos. Calidad En La Educacion, 247276. Retrieved from http://www.cned.cl/public/Secciones/ SeccionRevistaCalidad/doc/59/cse_articulo694.pdf

González Tirados, M. R., \& González Maura, V. (2007). Diagnóstico de necesidades y estrategias de formación docente en las universidades. Revista Iberoamericana de Educación.

Hernández, R., Fernández, C., \& Baptista, P. (2014). Metodología de la investigación. Journal of Chemical Information and Modeling (Vol. 53). https://doi.org/10.1017/ CBO9781107415324.004

Hogan, T. (2004). Pruebas psicológicas: una introducción práctica. México: Manual Moderno.

Imbernon, F. (1989). La formacion inicial y la formacion permanente del profesorado. dos etapas de un mismo proceso. Revista Interuniversitaria de Formación Del Profesorado.

Larraín U., A. M., \& Gonzalez F., L. E. (2005). Formacion universitaria por competencias. Seminario Internacional CINDA « Curriculo Universitario Basado En Competencias» Universidad Dek Norte, Barranquilla-Colombia. 25 y 26 de Julio.

Lemaitre, M. J., Maturana, M., Zenteno, E., \& Alvarado, A. (2012) Cambios en la gestión institucional en Universidades, a partir de la implementación del Sistema Nacional de Aseguramiento de la Calidad: La experiencia Chilena. Calidad En La Educación, 36, 21-52. https://doi.org/10.4067/S0718-45652012000100001

López, I. G., García, D. M., España, U. D. C., \& Barco, C. B. (2018). La formación permanente como herramienta para mejorar la intervención del maestro de educación física con alumnado con discapacidad Lifelong learning as a tool to improve physical education teachers 'intervention with students with disabilities, 2041, 118-122.

Messick, S. (1989). Validity in educational measurement. In Educational Measurement.

Ministerio de Educación. (2004). Reglamento sobre Evaluación docente. Departamento Jurídico.

Miranda Jaña, C., \& Rivera Rivera, P. (2009). Formacion Permanente De Profesores: ¿Quien Es El Formador De Formadores? Estu- dios Pedagógicos (Valdivia). https://doi.org/10.4067/S071807052009000100009

Montecinos, C., Rittershaussen, S., Solís, M. C., Contreras, I., \& Iglesias, J. (2010). Formacion de profesores basada en estandares: una propuesta para su evaluación. Assessment.

Norma, T., Materia, E. N., \& Municipal, D. E. G. (2016). Biblioteca del Congreso Nacional de Chile - www.leychile.cl - documento generado el 12-Mar-2016, 121-127. https://doi.org/10.1145/ 1542130.1542154

Otzen, T., \& Manterola, C. (2017). Técnicas de Muestreo sobre una Población a Estudio. International Journal of Morphology. https:/ /doi.org/10.4067/S0717-95022017000100037

Pedraja-Rejas, L. M., Araneda-Guirriman, C. A., Rodríguez-Ponce, E. R., \& Rodríguez-Ponce, J. J. (2012). Calidad en la Formación Inicial Docente: Evidencia Empírica en las Universidades Chilenas. Formación Universitaria. https://doi.org/10.4067/S071850062012000400003

Pedraz, M. V., Paz, M., \& Polo, B. (2014). Evolución de los planes de estudio de Educación Física y Ciencias de la Actividad Física en la Universidad de León: estudio de un (fra)caso Evolution of the syllabi in physical education and physical activity sciences at the University of León: a (failure, 26, 101-107.

Rodríguez Martínez, C. (Coord. . (2014). Revista Interuniversitaria de formación del profesorado. Revista Interuniversitaria de Formacion Del Profesorado. https://doi.org/10.1063/1.3080751

Rosas Morales, M., Backhoff Escudero, E., \& Larrazolo Reyna, N. (2000). Nivel de dificultad y poder de discriminación del Examen de Habilidades y Conocimientos Básicos (EXHCOBA). REDIE: Revista Electrónica de Investigación Educativa.

Rosas, R., Medina, L., Meneses, A., Guajardo, A., Cuchacovich, S., \& Escobar, P. (2011). Construcción y validación de una prueba de evaluación de competencia lectora inicial basada en computador. Pensamiento Educativo: Revista de Investigación Educacional Latinoamericana, 48(1), 43-62. https://doi.org/10.7764/ PEL.48.1.2011.4

Ruffnelli, A. (2014). Dificultades de la iniciación docente: ¿iguales para todos? Estudios Pedagógicos (Valdivia), 40(1), 229-242. https://doi.org/10.4067/S0718-07052014000100014

Rufinelli, A. (2011). Docente En Chile/ : La Perspectiva De Los Profesores Principiantes, 118-154.

Santelices Etchegaray, M. V., \& Valenzuela Rojas, F. (2015). Importancia de las características del profesor y de la escuela en la calidad docente: Una aproximación desde la Teoría de Respuesta del Ítem. Estudios Pedagogicos, 41(2), 233-254.

Sebastiani, E., Solá, J., Capell, M., Campos, J., \& Blásquez, D. (2009). Guía para la evaluación de competencias en ciencias de la actividad física y del deporte, 116. Retrieved from http://www.aqu.cat/ doc/doc_99076554_1.pdf

Serrano, G. P. (1989). La investigacion sobre el rendimiento academico y la calidad de la educacion. Revista de ciencias de la educación (madrid).

Sevillano, M. L. (2011). Didáctica en el núcleo de la pedagogía. Tendencias Pedagógicas, 18, 7-32.

Tobón, S., Rial Sánchez, A., Carretero, M. Á., \& García, J. A. (2014). Competencias, calidad y educación superior. Revista Mexicana de Investigación Educativa.

Urrejola, G., \& Williams, C. (2017). Impacto de dos instrumentos de evaluación en el rendimiento académico y su relación con los estilos de aprendizaje en estudiantes de Kinesiología. Revista de Educación En Ciencias de La Salud, 14(2), 7.

Urrutia Egaña, M., Barrios Araya, S., Gutiérrez Núñez, M., \& Mayorga Camus, M. (2015). Métodos Optimos Para Determinar Validez De Contenido. Revista Cubana de Educacion Medica Superior.

Vaillant, D. (2008). Algunos marcos referenciales para la evaluación del desempeño docente en América Latina. Revista Iberoamericana de Evaluación Educativa, 1(2), 7-22. https://doi.org/ 10.15366/riee

Vázquez, S. M., Biggio, M. N., \& García, S. M. (2013). Relaciones entre rendimiento académico, competencia espacial, estilos de aprendizaje y deserción. Revista Electronica de Investigacion Educativa

Villarroel, V. A., \& Bruna, D. V. (2017). Competencias Pedagógicas que Caracterizan a un Docente Universitario de Excelencia: Un Estudio de Caso que Incorpora la Perspectiva de Docentes y Estudiantes. Formacion Universitaria. https://doi.org/10.4067/ S0718-50062017000400008 